\title{
Extremely Low Magnification EBSD with an FE-SEM
}

\author{
Y. Yamamoto*, K. Ogura*, K. Kawauchi*, M. Shibata*, N. Erdman**, and C. Nielsen** \\ * JEOL 1td., 3-1-2 Musashino, Akishima, Tokyo, Japan \\ ** JEOL USA, Inc. 11 Dearborn Rd, Peabody, MA 01960, U.S.A.
}

Electron Backscattered Diffraction (EBSD) with an SEM has recently become very popular for the crystal orientation analysis of materials. It is quite useful to analyze tiny crystals at rather high magnification from several hundreds up to several tens thousands times. On the other hand, for the analysis of very large crystals with a size of several hundreds micrometers, a very low magnification SEM/EBSD is required. However, the very low magnification SEM/EBSD is somewhat problematic, because of the distortion of SEM images due to a large tilting angle of specimens. Recently, we have developed an FE-SEM (JSM-7001F/TTL), which resolved the image distortion problem at low magnification. SEM/EBSD with this JSM-7001F/TTL is significantly less affected by the image distortion even in the magnification range of less than one hundred times.

JSM-7001F/TTL equipped with an EBSD system (TSL OIM ${ }^{\mathrm{TM}}$ ) was used to investigate a sample of polysilicon for solar cell application. The JSM-7001F/TTL is a high-resolution Schottky FEGSEM. For high-magnification imaging, an electron probe is focused to a specimen by the objective lens (OL). On the other hand, when the low magnification imaging is necessary, the electron probe is focused to the specimen using the Aperture Angle Control Lens (ACL) instead of the OL. In this Low Magnification (LM) mode, the working distance between the ACL to the specimen is much longer as compared to using the OL. Furthermore, the aperture angle of the electron probe becomes much smaller as well. As the result, the LM mode of the JSM-7001F/TTL has an enormous depth of field without image distortion, thus allowing low-magnification SEM/EBSD with minimal artifacts. Fig. 1 shows a comparison between the conventional imaging mode and the LM mode. Fig. 2 shows an image comparison at ten times of the magnification between the conventional imaging mode and the LM mode using a 70 degree tilted metal grid.

Fig. 3 shows an optical microscope image of solar cell polysilicon with the size of $30 \mathrm{~mm}$ square. The area marked with a black rectangle line was used for SEM/EBSD. The actual SEM magnification for SEM/EBSD was $\mathrm{x} 12$. The analysis was performed at $20 \mathrm{kV}, 5 \mathrm{nA}$ of electron probe current, working distance $25 \mathrm{~mm}, 70$ degree of specimen tilt, and 100 micron EBSD measurement step. Fig. 4 shows results of low magnification EBSD, such as an image quality (IQ) map, and an inversed pole figure (IPF) maps. These results demonstrate that the LM mode of the JSM-7001F/TTL is suitable for the extremely low magnification SEM/EBSD applications. 


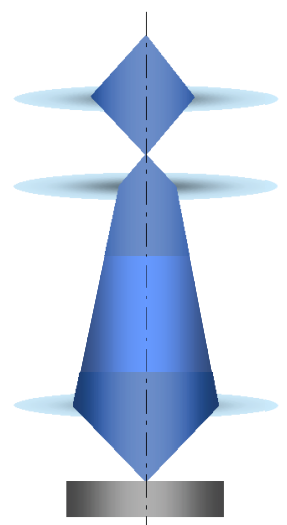

Normal mode

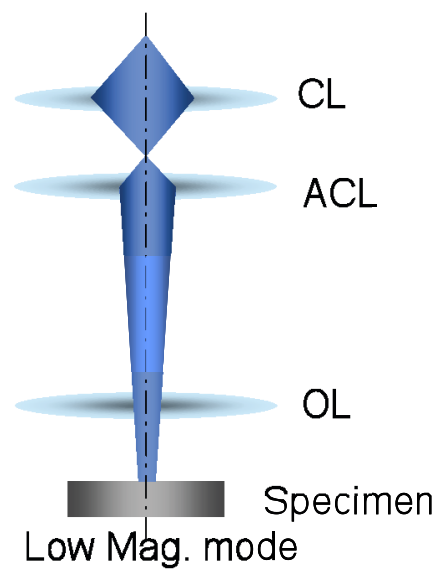

Fig. 1: Electron optics of JSM-7001F/TTL

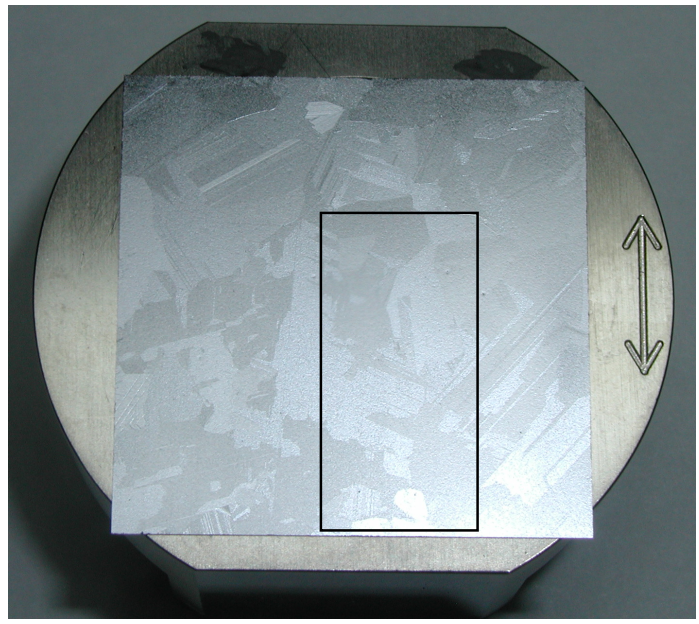

Fig.3: Polysilicon for solar cell

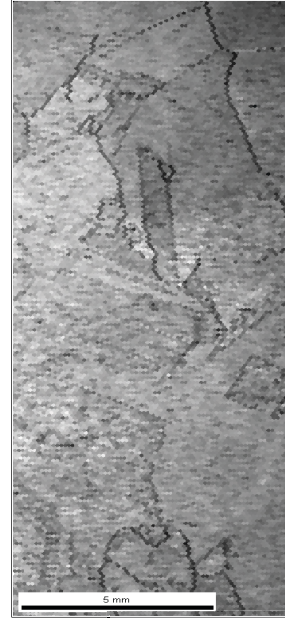

IQ

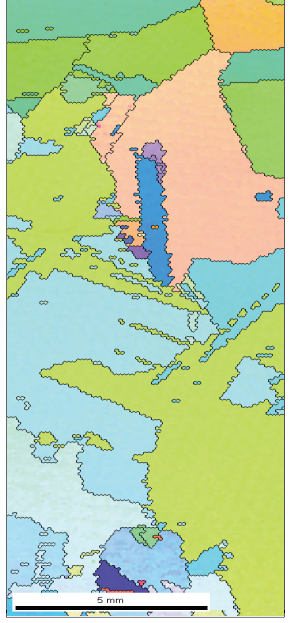

ND

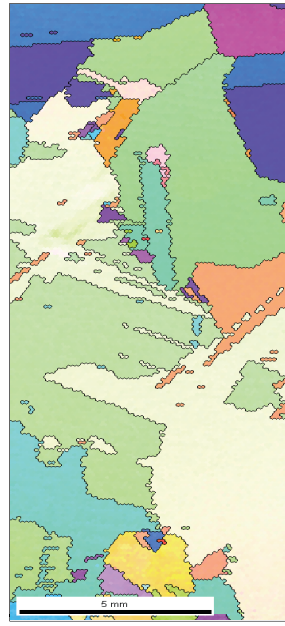

$\mathrm{RD}$

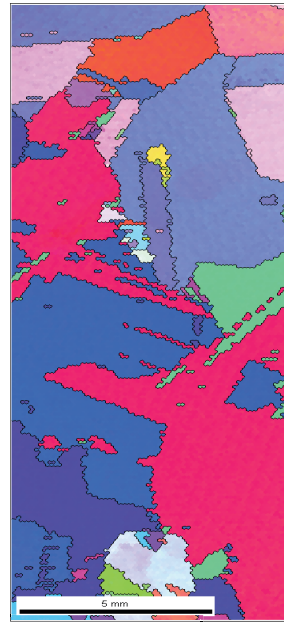

TD

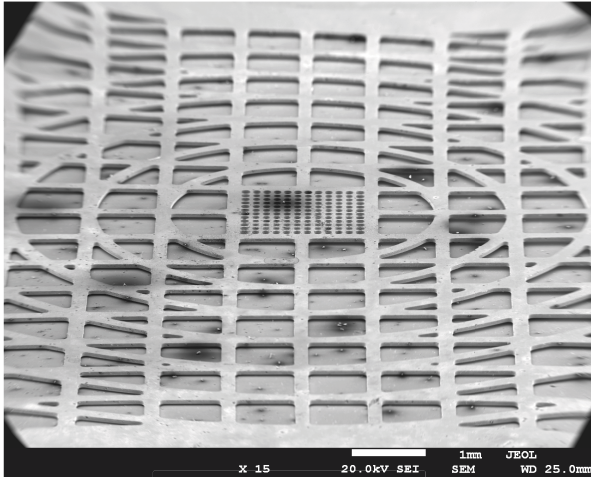

Normal mode

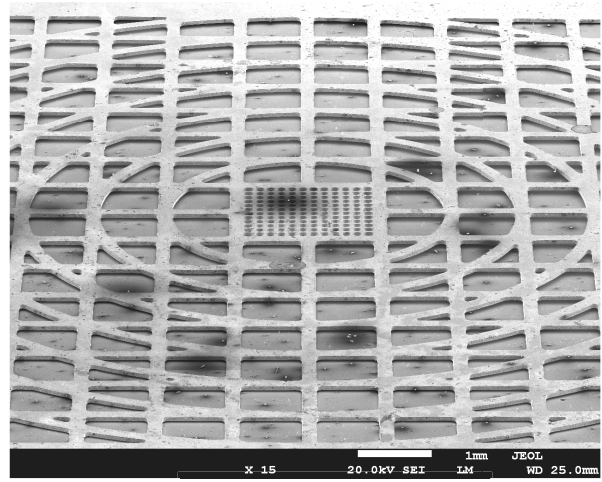

Low Mag. mode

Fig.2: SEM image of a $\mathrm{Cu}$ grid

Fig. 4: An image quality map and inverse pole figure maps 